\title{
Optical spectroscopic observations of the Be/X-Ray binary A0535+262/V725 Tau during the giant outburst in 2009
}

\author{
Yuuki Moritani ${ }^{1}$, Daisaku Nogami ${ }^{2}$, Atsuo T. Okazaki ${ }^{3}$, \\ Akira Imada $^{4}$, Eiji Kambe ${ }^{4}$, Satoshi Honda ${ }^{2}$, Osamu Hashimoto ${ }^{5}$ \\ and Kazuhide Ichikawa ${ }^{1}$ \\ ${ }^{1}$ Department of Astronomy, Kyoto University, Sakyo-ku, Kyoto 606-8502, Japan \\ email: moritani@kusastro.kyoto-u.ac.jp \\ ${ }^{2}$ Kwasan Observatory, Kyoto University, Yamashina-ku, Kyoto 607-8471, Japan \\ ${ }^{3}$ Faculty of Engineering, Hokkai-Gakuen University, Toyohira-ku, Sapporo 062-8605, Japan \\ ${ }^{4}$ Okayama Astrophysical Observatory, National Astronomical Observatory of Japan, \\ 3037-5 Honjo, Asakuchi, Okayama 719-0232, Japan \\ ${ }^{5}$ Gunma Astronomical Observatory, Takayama-mura, Gunma 377-0702, Japan
}

\begin{abstract}
A giant outburst occurred in A0535+262/V725 Tau in November 2009, which lasted approximately 30 days. We carried out spectroscopic monitoring at OAO and GAO from November 2009 to March 2010, from before the giant outburst to the rising phase of the normal outburst which occurred after the next periastron. The obtained H-alpha, H-beta and He I emission lines exhibited drastic profile variability during the observations.
\end{abstract}

Keywords. binaries: spectroscopic, stars: emission-line, Be, stars: individual (A0535+262)

\section{Introduction and Observation}

$\mathrm{Be} / \mathrm{X}$-ray binaries, which dominate the majority of high-mass X-ray binaries, consist of a Be star and a compact object, usually a neutron star (NS). The X-ray activity of Be/Xray binaries is divided into three states with respect to the luminosity; quiescent $\left(L_{X} \lesssim\right.$ $10^{36}$ ergs $\left.^{-1}\right)$, normal outburst $\left(L_{X} \sim 10^{36-37}\right.$ ergs $\left.^{-1}\right)$, and giant outburst $\left(L_{X} \gtrsim 10^{37}\right.$ $\left.\mathrm{ergs}^{-1}\right)$. These outbursts occur due to mass transfer from the Be disk, the geometrically thin circumstellar envelope formed by the outflow $(\lesssim 1 \mathrm{~km} / \mathrm{s})$ in the equatorial region and the rapid rotation (at 70-80\% of the critical speed), to the neutron star [Okazaki \& Negueruela (2001), Negueruela \& Okazaki (2001)].

$\mathrm{A} 0535+262 / \mathrm{V} 725 \mathrm{Tau}\left(P_{\text {orbital }}=110.2\right.$ days, e 0.47$)$ is one of the best studied Be/Xray binaries since its discovery [Rosenberg et al. (1975), Coe et al. (1975), Finger et al. (1994), Moritani et al. (2010)]. In November/December 2009, a giant outburst occurred in A0535+262 for the first time since 2005 [Sugizaki et al.(2009)]. Swift/BAT team reported that it reached more than 3 times of the Crab in the $15-50 \mathrm{keV}$ band. After the next periastron, in March 2010, a normal outburst occurred.

Optical spectroscopic observations of A0535+262 were carried out from November 2009 to March 2010 at the Okayama Astrophysical Observatory (OAO) with a $188 \mathrm{~cm}$ telescope equipped with HIDES (High Dispersion Echelle Spectrograph), and at Gunma Astronomical Observatory (GAO) with a $1.5 \mathrm{~m}$ telescope equipped with GAOES (GAO Echelle Spectrograph). The typical wavelength resolution $R$ and the signal to noise ratio $S / N$ of our data around $\mathrm{H} \alpha$ are $R \sim 30000-60000$ and $S / N \gtrsim 100$. The obtained data were reduced in the standard way using IRAF (see http://iraf.noao.edu) echelle package. 

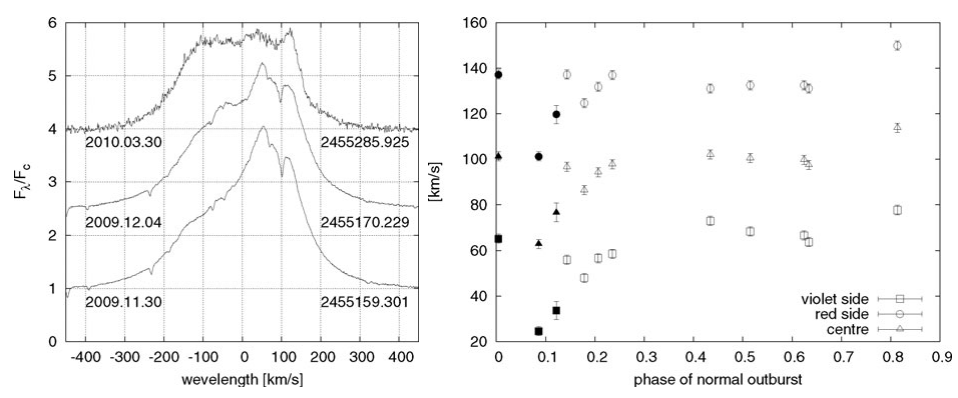

Figure 1. Left: representative $\mathrm{H} \alpha$ line profiles. Right: radial velocity of redshifted double-peaked component in $\mathrm{H} \alpha$ line profiles.

\section{Results and Conclusion}

The representative spectra of $\mathrm{H} \alpha$ obtained during the monitoring are shown in Fig. 1. For reasons of clarity, the spectra have linear offsets in the vertical axis from each other. The observation date and HJD of mid exposure time are written below each profile on the left and the right side, respectively.

Balmer and He I emission lines have changed drastically. The absolute value of equivalent width $(\mathrm{EW})$ around the giant outburst were highest in the last five years; $\mathrm{EW}(\mathrm{H} \alpha)$ $\sim-18 \AA, \operatorname{EW}(\mathrm{H} \beta) \sim-3 \AA$ and $\operatorname{EW}(\mathrm{He} \mathrm{I} \lambda 5875) \sim-2 \AA$. During the observations, emission line profiles had the redshifted $(\sim 100 \mathrm{~km} / \mathrm{s})$ enhanced components (double peaked in $\mathrm{H} \alpha$, and single peaked in other lines). The enhanced component also showed variability along with the X-ray flux.

Optical emission lines come from the Be disk mainly, and therefore observed line profile variability implies that the Be disk changed during the giant outburst and the next normal outburst. However, the contribution of the accretion disk around the NS may not be negligible, since it may grow enough. We then measured the radial velocity of the double-peak component in the red part of $\mathrm{H} \alpha$ to check which is the emitter, the Be disk or the disk around the NS (Fig. 1). As a result, the radial velocity decreased around the periastron. The geometry presented in Coe et al. (2006) indicates that the Be star goes back away to an observer at periastron passage. Considering this fact, it is plausible that the enhanced component comes from not the accretion disk but the Be disk.

After the giant outburst, a couple of normal outburst outbursts have occurred [CameroArranz et al.(2010)] The Be disk of A0535+262 is still active according to its EW, and then the next several periastron passage should be a good chance to chase the interaction of the Be disk and the NS.

\section{References}

Camero-Arranz, A., Finger, M. H., Wilson-Hodge, C., \& Jenke, P. 2010, The Astronomer's Telegram 2705, 1

Coe, M. J., Carpenter, G. F., Engel, A. R., \& Quenby, J. J. 1975, Nature, 256, 630

Coe, M. J., Reig, P., McBride, V. A., Galache, J. L. et al. 2006, MNRAS, 368, 447

Finger, M. H., Cominsky, L. R., Wilson, R. B., Harmon, B. A. et al. 1994, in: S. Holt \& C. S. Day (eds.), The Evolution of X-ray Binariese, AIP-CP 308, p. 459

Moritani, Y., Nogami, D., Okazaki, A. T., Imada, A . et al. 2010, MNRAS, 405, 467

Negueruela, I. \& Okazaki, A. T. 2001, A\& $A$ 369, 108

Okazaki, A. T. \& Negueruela, I. 2001, A\&A A, 377, 161

Rosenberg, F. D., Eyles, C. J., Skinner, G. K., \& Willmore, A. P. 1975, Nature, 256, 628

Sugizaki, M., Mihara, T., Kawai, N., Nakajima, M. et al. 2009, The Astronomer's Telegram 2277,1 